\title{
Standardization Works for Security Regarding the Electromagnetic Environment
}

\author{
Tetsuya Tominaga \\ NTT Energy and Environment Systems Laboratories \\ 3-9-11, Midori-cho, Musashino-shi, Tokyo, 180-8585
}

\begin{abstract}
Telecommunication functions of electronic devices have been and will continue to increase. The so called smart community, a society in which more advanced communications technology is used, will enable life to be increasingly convenient. Thus, telecommunications will become more and more important. However, when such functions become unavailable for some reason, it will negatively impact society. Therefore, device robustness and information leakage are serious issues that need to be addressed. Security regarding electromagnetic waves has been extensively studied in terms of electromagnetic compatibility. In particular, high power electromagnetic phenomena and information leakage due to electromagnetic waves have been discussed in IEEE EMC TC5, ITU-T SG5 and IEC SC77C. In this presentation, an overview of the results, trends, and future works are discussed. Recently developed recommendation ITU-T K.84 (test methods and guide against information leaks through unintentional EM emissions), a leakage mechanism, and protection methods are also discussed.
\end{abstract}

